\title{
MT-ATP6-related mitochondrial spastic paraplegia
}

INSERM

\section{Source}

INSERM. (1999). Orphanet: an online rare disease and orphan drug data base. MT-ATP6related mitochondrial spastic paraplegia. ORPHA:320360

MT-ATP6-related mitochondrial spastic paraplegia is a rare, genetic, complex hereditary spastic paraplegia disorder characterized by adulthood-onset of slowly progressive, bilateral, mainly lower limb spasticity and distal weakness associated with lower limb pain, hyperreflexia, and reduced vibration sense. Axonal neuropathy is frequently observed on electromyography and nerve conduction examination. 